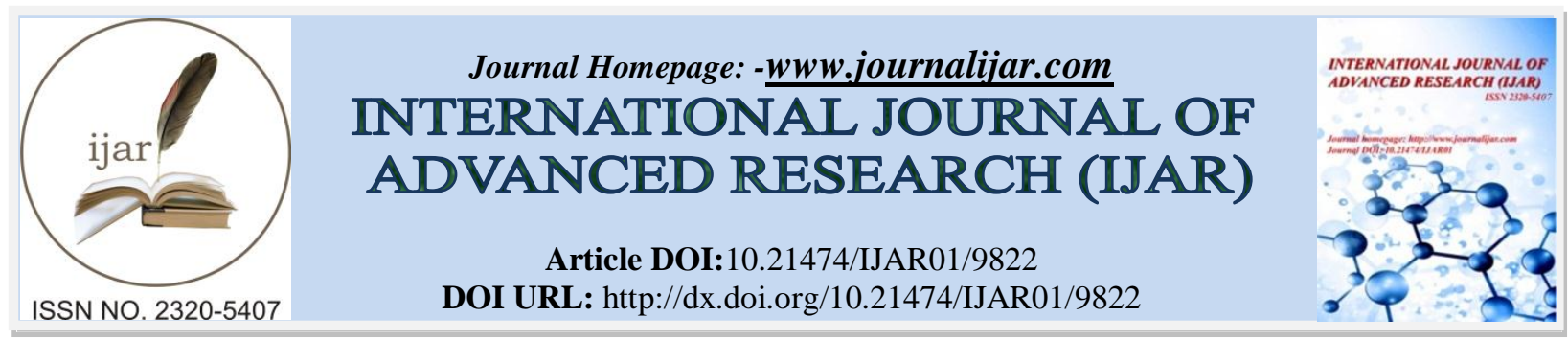

RESEARCH ARTICLE

\title{
MICROBIOLOGICAL REVIEW OF PLEURAL FLUID SAMPLES A RETROSPECTIVE STUDY
}

Dr. Tazammul Hussain ${ }^{1}$, Dr. M.V.Mulay ${ }^{2}$, Dr. A. S.Wyawahare ${ }^{3}$ and Dr. Ishan Pandita ${ }^{1}$.

1. PG Resident, Department of Microbiology, MGM medical college \& Hospital,Aurangabad,Maharashtra

2. Professor \& Head, Department of Microbiology,MGM medical college \& Hospital,Aurangabad, Maharashtra

3. Professor, Department of Microbiology, MGM medical college \& Hospital ,Aurangabad, Maharashtra.

\section{Manuscript Info}

\section{Manuscript History}

Received: 06 August 2019

Final Accepted: 08 September 2019

Published: October 2019

Key words:-

Plerual Fluid, Microbial etiology,

\begin{abstract}
Since the advent of antibiotics the microbial etiology of pleural space infections has changed. This can be due to patients related factors such as trauma, underlying disease or surgical procedures, or other reason can be due to methodological factors viz specimen collection, transport and its culture. For these reasons, several studies have found disagreeing findings in the spectrum of pathogens causing pleural space infection. The present study was carried out in department of Microbiology, MGM Medical College, Aurangabad, Maharashtra, India with the aim to review the microbiological profile of pleural fluid samples and to observe the antibiogram of the isolates in pleural fluid.
\end{abstract}

Copy Right, IJAR, 2019,. All rights reserved.

\section{Introduction:-}

The term pleural effusion is used to describe a non-purulent serous effusion which sometimes forms in pneumonia, tuberculosis, malignant diseases, or pulmonary infarction (embolism). ${ }^{[1]}$ The most frequent manifestation of intrathoracic disease are pleural effusion and empyema, which are associated with poor outcome of affected patients. Transudative pleural effusion are due to Congestive cardiac failure, liver cirrhosis or systemic diseases, whereas exudative pleural effusions occur may be due to malignancy or due any inflammatory process ${ }^{[2]}$. Complications of pneumonia usually give rise to empyema but it may also arise due to infections at other sites. ${ }^{[2]}$ Since the introduction of antibiotics in 1970s the microbial aetiology of pleural space infections has changed, this can be due to patients related factors such as trauma, underlying disease or surgical procedures, or other reason can be due to methodological factors viz specimen collection, transport and its culture. For these reasons, several studies have found disagreeing findings in the spectrum of pathogens causing pleural infection. ${ }^{[2,3,4,5,6,7]}$ So to know the micrbiological profile of pleural space infections in MGM Medical college, Aurangabad, Maharashtra, India, this study was conducted over a period of 12 months.

\section{Material and Methods:-}

This was a retrospective observational study conducted in the department of microbiology after the approval of the institution ethical committee.

The pleural fluid samples collected aseptically by throracocentesis from patients of various departments in hospital and received in microbiology laboratory for bacteriological culture from IPD patients and for mycobacterium detection from opd patients in RNTCP center were included in the study which were received between June 2018 to 
May 2019. Gram staining was done for the samples received for bacteriological culture as per standard protocol. ${ }^{[8]}$ For identification of the bacterial isolates and antimicrobial susceptibility testing, Vitek 2 compact system (Biomeriux) was used. And for pleural fluid specimen collected from OPD patients of suspected tuberculosis in RNTCP, initially screened with flurochrome staining method for microscopy, as this method more sensitive and quicker than the conventional Ziehl-Neelsen staining. ${ }^{[1]}$ Fluorochrome staining done as per the guidelines by RNTCP. ${ }^{[9]}$ All the samples of Pleural fluid received in RNTCP were processed in CBNAAT (Cartridge Based Nucleic Acid Amplification Test) for Mycobacterium tuberculosis detection.

\section{Result and Discussion:-}

Over a period of 12 months 104 samples were received for bacteriological culture and 213 samples were received in RNTCP for mycobacterium tuberculosis detection on CBNAAT in microbiology laboratory. Out of the total 104 samples only $4(3.8 \%)$ gave positivity on bacteriological culture and out of total 213 samples tested in CBNAAT only $19(9 \%)$ were positive for Mycobacterium tuberculosis.

Maximum patient for bacteriological culture were in the age group of more than 60 years 30 (29\%) followed by4049 yr age group $16(15 \%)$ [Fig 1]. Male to female ratio was 2:1.

Direct detection of microorganisms by Gram stain was $11 \%$ and by fluorochrome staining, for Mycobacterium, was $9 \%$.

For bacteriological culture, equal number of gram positive and gram negative organisms were isolated. Among the gram negative organisms Salmonella paratyphi A and Klebsiella pneumonia was isolated. Salmonella paratyphi A was an Extended spectrum beta lactamase (ESBL) producer and Klebsiella pneumoniae was a multidrug resistant isolate. Among gram positive organism Staphylococcshaemolyticus and Staphylococcus aureus was isolated [Table 1].

For mycobacteriological study Nineteen (9\%) patients showed infection with Mycobacterium tuberculosis as detected by CBNAAT in RNTCP and none of them was a multidrug resistant isolate. All the patients included in this study were HIV sero-negative.

Antibiotic susceptibility test results were also compiled for both gram negative and gram positive organisms. Among the gram negative organisms 100\% resistance was observed for Ciprofloxacin, Gentamicin , Amikacin and Sensitive to Cotrimoxazole (50\%) and meropenem (50\%).

$50 \%$ of the isolate showed extended spectrum beta lactamases (ESBL) production.

Among the gram positive organisms $100 \%$ resistance was noted for penicillin and cotrimoxazole. All the isolates were sensitive to Vancomycin, Linezolide, Clindamycin and Gentamicin. No methicillin-resistant Staphylococcus aureus was isolated. (Fig 2)

Among the isolated Mycobacterium tuberculosis, none of the isolate was multidrug resistant. All the patients screened for Mycobacterium tuberculosis were HIV sero-negative. Fluorescent microscopy screening of samples had 19 smear positive for mycobacterium bacilli that is same as the number of CBNAAT positive samples.

In the present study, conducted in a tertiary care teaching hospital comprising of 104 samples for bacteriological culture, the percentage of positive cultures was $3.8 \%$. As in earlier studies, the microbiological diagnosis has shown variation. Walshe and colleagues have reported $3 \%$ positivity in pleural fluid culture ${ }^{[10]}$. On the other hand, high positive culture rates has been reported by various other studies. ${ }^{[2]}{ }^{[7]}$. The reason for this disparity in rates of positive culture can be either due to differences in techniques, antibiotic use or due to prevalence of effusions caused by infectious processes ${ }^{[3]}$. In our study, most of the patients had already received antibiotic before collection of pleural fluid samples.

The high incidence of pleural effusion in the age group of more than 60 years is consistent with the findings in the earlier study by $\mathrm{J}$ Porcel et al ${ }^{[2]}$. 
In our study we report equal numbers of the gram positive isolate and the gram negative isolate. Among the gram positive isolates Coagulase negative staphyolococcus $(25 \%)$ was isolated which was resistant to cefoxitin. Staphylococus aureus (25\%) was isolated which was cefoxitin sensitive. The Gram positive organisms were $100 \%$ resistant to penicillin and cotrimoxazole. SoodAnuradha et al reported resistant to penicillin (57.1\%) and clindamycin $(42.8 \%)^{[7] .}$ All the gram positive isolates were sensitive to vancomycin, linezolide, clindamycin and Gentamicin. Similar findings were reported by Soodanuradha et al ${ }^{[7]}$. In our study none of the isolate was Methicillin resistant Staphylococcus aureus(MRSA). This is in contrast to the findings of Soodanuradha et al where they have reported $21.4 \%$ of the isolates being MRSA ${ }^{[6]}$. Prevalance report from different parts of the country varies. $^{[13]}$

Here we report a case of Salmonella paratyphiA (25\%) isolated from a young female patient, the organism was an ESBL producer and it was sensitive to cotrimoxazole and resistant to ciprofloxacin. These findings in our study is not in agreement with the study of P.mathur et al. They reported Salmonella paratyphi A from a case of child less than 10 year of age and the reported organism was sensitive to ciprofloxacin and resistant to cotrimoxazole [11]. Other Gram negative isolate was Klebsiellapneumoniae(25\%) which was resistant to amoxicillin-clavulanic acid, ampicillin, piperacillin-tazobactam, cefuroxime, ceftriaxone, cefepime, meropenem, amikacin, gentamicin. ciprofloxacin, cotrimoxazole. Intermediate susceptible to tigecycline and imipenem. Highly Susceptible to colistin . Whereas in the study conducted by Soodanuradha et al, the gram negative isolates were resistant to cotrimoxazole, and amoxicillin-clavulanic acid. They reported gram negative isolates were sensitive to Gentamicin and imipenem. ${ }^{[7]}$

CBNAAT (cartridge based nucleic acid amplification test) is a recent introduced polymerase chain reaction (PCR) based method for the detection of tuberculosis. It also detects rifampicin resistance by targeting rpoB gene in mycobacteria. It is a mycobacterium tuberculosis specific automated method providing results within 100 minutes. ${ }^{[12]}$ In our study, Mycobacterium tuberculosis was isolated in $9 \%$ of patients, no Multidrug resistant mycobacterium tuberculosis was isolated. As per the study of Acharya et al, out of 26 patients, $73 \%$ were detected with tuberculosis in pleural fluid ${ }^{[5]}$. Gupta et al reported $13.8 \%$ positivity in pleural fluid analysis. ${ }^{[14]}$

Limitations of our study: because of the variation in the clinician's requisitions, all pleural effusions could not be categorized as transudate or exudate. Another important factor for the decrease in the yield of culture is that the patients have received antibiotics before their thoracocentesis.

\section{Conclusion:-}

Bacteria are evolving constantly with new mechanisms of antibiotic resistance limiting the use of currently available antibiotics. To get over this limitation, active surveillance program for antibiotic use \& correct implementation of the antimicrobial stewardship program must be ensured. CBNAAT detects MTB with greater efficacy and it is a useful tool for the diagnosis for tuberculosis in the short span of time to start the necessary treatment.

\section{Acknowledgement:}

We are thankful to the ethical committee for permitting us to conduct this study.

\section{Conflict of interest: \\ None}

Financial assistance :

None. 
Fig 1:-Distribution of patients as per the Age group

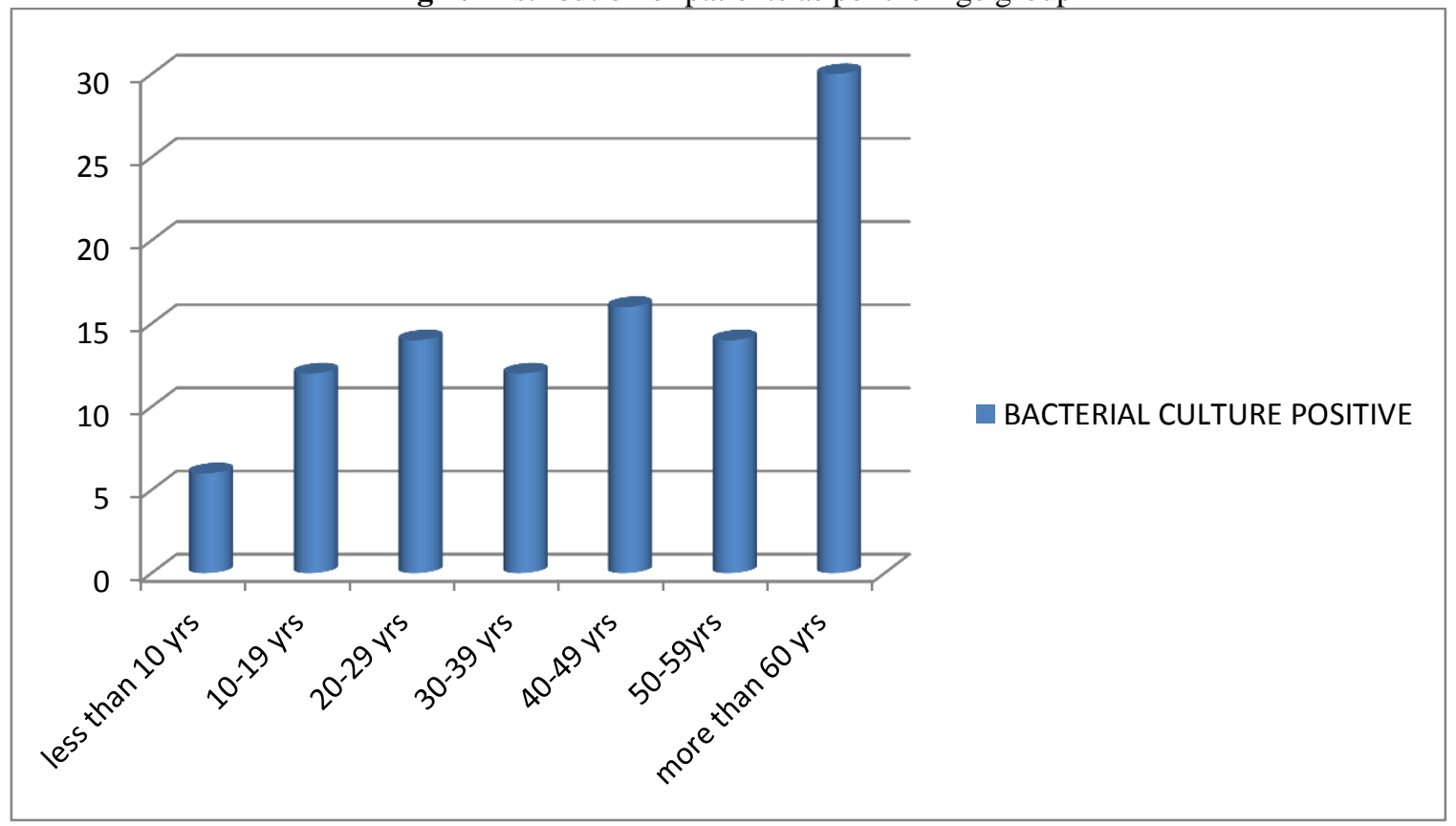

Table 1:-Spectrum of various organisms isolated

\begin{tabular}{|l|l|l|l|}
\hline Organism isolated from culture & No. of Isolates [4] & $\%$ of isolates & Special characters \\
\hline Staphylococcus aureus & 1 & $25 \%$ & MSSA \\
\hline Coagulase negative Staphylococcus & 1 & $25 \%$ & MRCONS \\
\hline Salmonella paratyphi A & 1 & $25 \%$ & ESBL \\
\hline Klebsiella pneumoniae & 1 & $25 \%$ & MDR \\
\hline
\end{tabular}

Fig 2:-\% Resistance of gram positive isolates

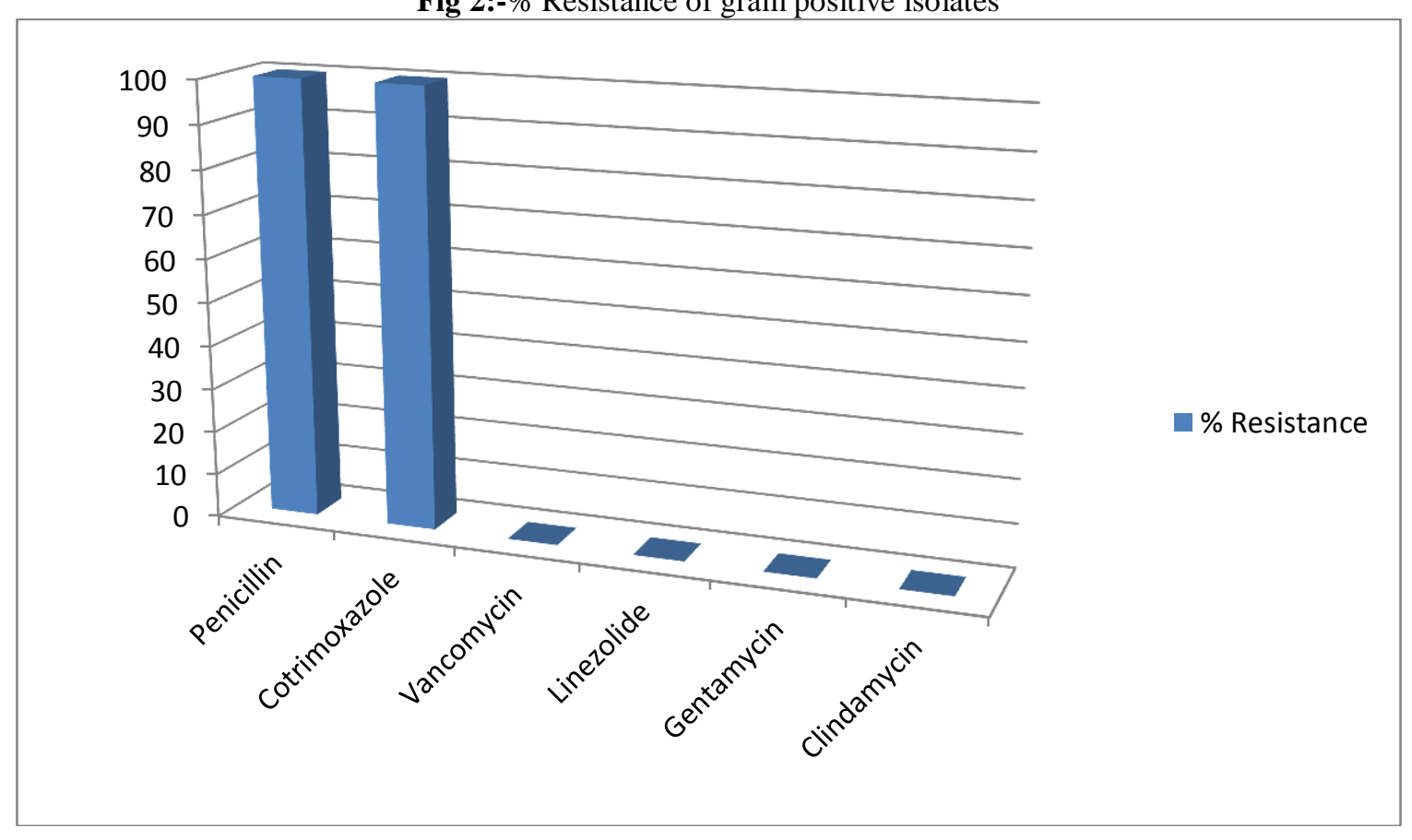




\section{References:-}

1. District laboratory practice in tropical countries. $2^{\text {nd }}$ ed update part 2 Monica Cheesbrough

2. J Porcel, P Vázquez, M Vives. Pleural space infections: Microbiologic and fluid characteristics in 84 patients. The internet journal of pulmonary medicine. 2002 Volume 3 Number 1.

3. Terrance W Barnes, Timothy I; Low yield of microbiologic studies on pleural fluid specimens CHEST /127/3/MARCH,2005, 916-921

4. Amit banga, G C Khilani; A study of empyema thoracis and role of inntrpleural streptokinase in its management ,BMC Infectious diseases 2004, 4:19

5. PreetamRajgopal Acharya, Kusum V Shah; Empyema thoracis :a clinical study ,Annals of Thoracic medicineVol2, Issue 1, January-March 2007.

6. KY tsang, WS Leung; Complicated parapneumonic effusion and empyema thoracis: microbiology and predictors of adverse outcomes ,Hong Kong Med J Vol 13 No3 June 2007.

7. Sharma ShaifaliSoodanuradha; Microbiological analysis of pleural fluids : a study frpm Dr. Rajendra Prasad government medical college, Kangra at Tanda (Himachal Pradesh, India) ,Indian J Med Research \& Phar Sci April 2018; 5(4).

8. Bailey \& Scott's Diagnostic Microbiology $14^{\text {th }}$ ed, Elsevier, Ch 6 p 76

9. RNTCP Fluorescence microscopy SOP final Draft version 31-3-07.

10. Walshe AD, Douglas JG, Kerr KM, et al. An audit of the clinical investigation of pleural effusion. Thorax 1992 ; $47 ; 734-737$.

11. P mathur ,Renuka, L Simpson. Indian paediatrics 2003; 40: 252-254.

12. R Dewan, S Anuradha; Role of cartridge-based nucleic acid amplification test (CBNAAT) for early diagnosis of pulmonary tuberculosis in HIV, JIACM 2015; 16(2): 114-7

13. Peter M Hawkey and Annie M Jones. The changing epidemiology of resistance Journal of antimicrobial chemotherapy (2009), 64, Suppl, 1, i3-i10

14. Mahesh gupta, gopalpurohit, sunilvyas. Prevalance of positivity of CBNAAT in extrapulmonary tuberculosis. European respiratory journal 2018 52: Suppl.62, PA2756. 